\title{
Métaphores terminologiques : fonctions et statut dans les langues de spécialité
}

\author{
Rossi, Micaela \\ Université de Gênes \\ micaela.rossi@unige.it
}

\section{Les recherches sur la métaphore en terminologie, entre linguistique et épistémologie}

Le rôle fondamental des métaphores dans la création des termes et dans la conceptualisation des domaines de spécialité est un phénomène amplement reconnu et étudié, notamment au cours des dernières décennies : les études des linguistes et terminologues (entre autres, les essais de Kocourek, 1991, puis d'Assal 1994 et plus récemment de Cortès 2003 et d'Oliveira, 2009), mais aussi des philosophes des sciences et des épistémologues (des analyses de Kuhn et Boyd 1979, aux essais plus récents de Schlanger 1995 et de Schlanger et Stengers 1991) mettent en évidence tout particulièrement la fonction heuristique de la métaphore, figure de concept qui, par le biais d'une prédication allotopique, permet de déclencher une interaction conceptuelle entre deux domaines notionnels, entraînant à son tour une nouvelle vision du concept-cible (nous renvoyons à ce propos aux ouvrages de Michele Prandi, dans la bibliographie finale ${ }^{1}$ ).

Les terminologues en particulier, notamment à partir des années 90, consacrent une attention nouvelle aux mécanismes de terminogenèse à base métaphorique, considérant essentiellement l'aspect dénominatif de la figure, utilisée comme ressort pour la formation de nouveaux termes facilement compréhensibles et accessibles aux usagers. Dans ces études, la métaphore est surtout considérée dans sa dimension de catachrèse, étiquette appliquée sur un nouveau concept, comblant un vide dénominatif, en ligne avec la tendance uninotionnelle et universaliste qui caractérisera la terminologie jusqu'à la fin des années 90 .

Les épistémologues, en revanche, s'intéressent plutôt au pouvoir heuristique de la métaphore, à son potentiel en termes de modélisation de nouvelles théories, comme le prouvent, entre autres, les essais de Schlanger (1995); dans ces études, la métaphore est surtout une interaction de concepts qui apporte un éclairage nouveau sur les découvertes techniques et scientifiques, dans la mesure où elle fournit aux usagers un accès immédiat aux notions abstraites qui caractérisent souvent les sciences, un accès qui passe par l'analogie et l'interaction des concepts et qui rend plus transparente la nouvelle notion.

Dans les langues spécialisées, la métaphore accomplit donc une fonction explicative, mais également une fonction constitutive, notamment dans les cas de métaphores fondatrices de théories (par exemple, la théorie darwinienne caractérisée par la métaphore de la sélection naturelle, ou encore la théorie des cordes de Veneziano qui repose sur l'interaction entre le domaine de la physique et les instruments musicaux). Par le biais de ces métaphores, illustration du processus créatif de la pensée, les sciences se réapproprient leur rôle productif, celui d'imaginer des univers possibles (Montuschi, 2013), voire incohérents, qui sollicitent nos capacités d'inférence et de reconstitution de réalités potentielles :

Ce qui se produit est un phénomène que Koestler nomme bissociation : une synthèse immédiate entre deux zones ou matrices. Les deux matrices étaient séparées : soit dans l'espace des disciplines, soit même dans le temps. [...] Le saut dramatique qu'est le rapprochement est l'acte même de la créativité [...] L'activité métaphorique se 
présente comme la face verbale de la conceptualisation inventive (Stengers, 1995, p.80-87)

Ce n'est que pendant les années 2000 que les deux approches se rejoignent progressivement: la terminologie évolue vers un modèle de plus en plus textuel et sociolinguistique (comme en témoignent l'approche socioterminologique (Gaudin, 2003), la terminologie sociocognitive (Temmerman 2000), la théorie communicative (Cabré, 1999) et la terminologie culturelle (Diki-Kidiri, 2000). Ces approches appellent une réflexion plus ample sur l'ensemble des manifestations métaphoriques dans les contextes techniques et scientifiques, contribuant ainsi à une progressive prise de conscience de la multiplicité et de la complexité des réalisations de la métaphore en terminologie, dépassant la simple dimension épisodique de la catachrèse. Ce tournant fondamental est également stimulé par les avancées des disciplines connexes, telles que la linguistique textuelle, la pragmatique, la linguistique cognitive - dans ce sens l'apport de la théorie cognitive de l'interaction métaphorique dans les essais de Richards (1936), Black (1962) et notamment de Lakoff et Johnson (1980) s'avère fondamental : à la vision de la métaphore comme une simple étiquette - jusqu'alors l'apanage des terminologues - et à la vision de la métaphore comme outil fondateur de paradigmes scientifiques - jusqu'alors l'apanage des épistémologues - s'ajoute le concept d'isotopie cognitive métaphorique, interaction conceptuelle en mesure de générer diverses réalisations linguistiques- notamment lexicales.

La figure est ainsi finalement considérée dans sa complexité : loin d'être une simple catachrèse épisodique fondée sur une analogie référentielle pour combler des vides terminologiques, loin d'être uniquement une stratégie de légitimation de paradigmes épistémologiques dans l'histoire de la pensée scientifique, loin d'être une simple banalisation de modélisations cognitives universelles dans notre vie quotidienne, la métaphore dans les langues de spécialité est fondée sur des processus et des phénomènes complexes, multiformes, jouant des fonctions sémiotiques variables et différentes. Cette nouvelle conception de la complexité métaphorique dans les contextes spécialisés est évidente surtout dans les ouvrages récents, où les chercheurs soulignent notamment la variété des fonctions que la métaphore peut jouer dams les langues de spécialité, avec une attention particulière pour la fonction communicative de la métaphore - comme il ressort entre autres des essais de Collombat (2003) dans le sillage des études sur la vulgarisation scientifique de Jacobi (1987, 1999), ou, plus récemment, des études d'Oliveira (2009) sur les fonctions de la métaphore en cardiologie, ou du volume collectif dirigé par Dury, Maniez, Arlin et Rougemont (2009), où la métaphore est mise à l'épreuve de diverses approches méthodologiques et de divers domaines thématiques.

\section{Fonctions et nature sémiotique de la métaphore terminologique}

Diverses fonctions sont donc attribuées à la métaphore dans les langues spéciales. Oliveira (2009) en dresse une typologie sommaire dans son ouvrage sur la métaphore en cardiologie :

- Une fonction dénominative dans les cas où la métaphore - d'habitude une catachrèse isolée sert à combler un vide dénominatif dans un domaine spécialisé - c'est le cas par exemple de l'induit à cage d'écureuil dans la terminologie photovoltaïque ;

- Une fonction heuristique dans les cas où la métaphore joue le rôle de déclencheur pour établir des analogies et favoriser la compréhension de nouvelles théories ;

- Une fonction herméneutique, lorsque la métaphore favorise la compréhension immédiate et accessible de concepts scientifiques abstraits - Oliveira cite l'exemple du brevet novacor plus accessible dans sa forme métaphorique cour de lion ;

- divulgatrice, dans les cas où la métaphore joue un rôle explicatif (voir Jacobi, 1986, 1999), notamment pour les locuteurs profanes ;

- didactique, dans les cas où la métaphore a la fonction d'aider les apprenants dans un domaine spécifique, les aidant progressivement à passer du statut de profanes au statut de spécialistes. 
Dans les essais plus récents de Prandi (2012), la variété de la figure métaphorique concerne en revanche la nature sémiotique des expressions métaphoriques dans les textes de spécialité, allant de la simple catachrèse isolée, aux concepts cohérents et partagés, aux métaphores vives défiant nos conditions de cohérence ordinaires pour générer une projection fonctionnelle à la création de nouveaux paradigmes épistémologiques. La métaphore n'est ainsi ni soumise à la vision unitaire traditionnelle, ni contrainte à une évolution obligée (de la métaphore vive à la catachrèse, voir l'hypothèse de la carrière métaphorique de Bowdle et Gentner, 2005), évoluant vers une conception sémiotiquement plus complexe et structurée : la dénomination de métaphore terminologique renvoie alors à une pluralité d'emplois qui, loin d'être homogènes, diffèrent par leur comportement textuel et discursif, ainsi que par leur nature sémiotique.

Dans ce sillage, Prandi distingue trois statuts sémiotiques différents des métaphores terminologiques: dans un premier cas, plus fréquent dans les langages techniques à forte concentration de notions à référent concret, la métaphore a le statut de catachrèse sur la base d'une analogie formelle : c'est le cas par exemple du nain dans le langage technique des chemins de fer :

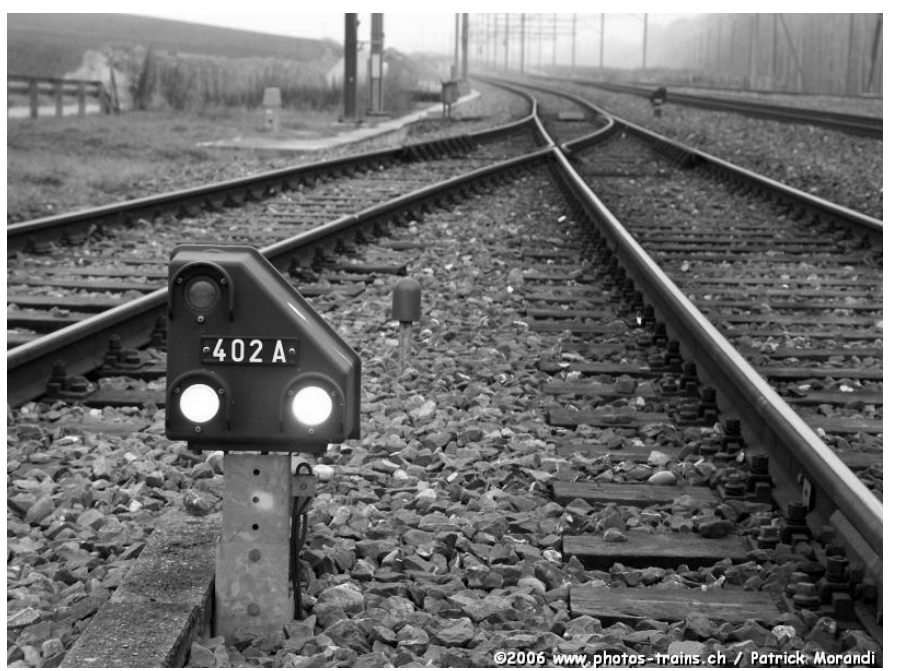

Figure 1 - Un nain

Dans ce cas, la métaphore a une fonction dénominative permettant de combler des lacunes dans un vocabulaire spécifique, par des dénominations accessibles et aisément compréhensibles; toutefois, le pouvoir heuristique de la figure reste limité au cas isolé, cristallisé dans l'analogie formelle.

Dans un deuxième cas de figure, la métaphore est exploitée comme un renvoi cognitif à une isotopie analogique partagée, fonctionnelle à la création de nouveaux termes: c'est le cas par exemple de l'isotopie anthropomorphique dans le langage de la dégustation du vin, dont la productivité dans diverses langues ressort du glossaire de l'AREV (Association des Régions Européennes Viticoles) reproduit ci dessous : 


\begin{tabular}{|c|c|c|c|c|}
\hline amabile & moelleux & semi dulce & lieblieche & medium sweet. \\
\hline corposo & $\begin{array}{l}\text { étoffé, plein, bien } \\
\text { en chair }\end{array}$ & robusto, carnoso & $\begin{array}{l}\text { korperreich, woll, } \\
\text { füllig }\end{array}$ & $\begin{array}{l}\text { robust, } \\
\text { bodied }\end{array}$ \\
\hline maturo & fait, mût, evolué & maduro & ausgebaut & mature, ripe wifle \\
\hline molle & moll & debil & weichlich & Flabby \\
\hline morbido & souple & morbido suave & $\begin{array}{l}\text { geschmeidig, } \\
\text { gefallig }\end{array}$ & supple morbido \\
\hline robusto & vin fort, puissant & $\begin{array}{ll}\text { vinho } & \text { robusto } \\
\text { vino } & \text { robusto, } \\
\text { fuette } & \end{array}$ & $\begin{array}{l}\text { machtiger, } \\
\text { wuchtiger Wein }\end{array}$ & $\begin{array}{l}\text { strong, powerfill } \\
\text { wine }\end{array}$ \\
\hline generoso & win généreux & wino generoso & $\begin{array}{l}\text { rassiger, edler, } \\
\text { nobel Wein }\end{array}$ & fill wine \\
\hline grossolano & vifl grossier & $\begin{array}{l}\text { vino grosero, } \\
\text { basto }\end{array}$ & plumper Wein & coarse witle \\
\hline magro & win maigre & vino pobre, & sutil dünner Wein & thin wine \\
\hline fiacco & vin plat & $\begin{array}{l}\text { vino insipido, } \\
\text { delgado }\end{array}$ & $\begin{array}{l}\text { fader, milder, } \\
\text { weicher, schaler, } \\
\text { abge } 3 \text { tandener, } \\
\text { glatt Wein }\end{array}$ & Flat, insipid wine \\
\hline vivace & win vivace & wino wivaz & lebendiger Wein & $\begin{array}{l}\text { vivacious wine, } \\
\text { lively }\end{array}$ \\
\hline
\end{tabular}

Figure 2 - Exemples tirés du glossaire AREV

De même, l'isotopie univers = personne est à la base de nombreuses métaphores terminologiques dans le langage de l'astrophysique, telles que générations d'étoiles, étoiles génitrices, pouponnière d'étoiles (Giaufret, Rossi, 2010)...

Dans le dernier cas de figure, la métaphore retrouve tout son pouvoir conflictuel au niveau de la pensée, nous obligeant ainsi à des conceptualisations nouvelles qui défient la cohérence du discours ordinaire nous sommes dans ce cas en présence de métaphores fondatrices de théories, telle que la métaphore des cordes dans la théorie astrophysique de Gabriele Veneziano (1968) qui joue sur l'analogie entre les particules élémentaires de l'univers et les cordes d'un violon, nous ouvrant de nouvelles perspectives sur un phénomène totalement abstrait, complexe, et dont nous n’avons aucune expérience sensible.

\section{Ancrage culturel des métaphores terminologiques : les problèmes de transfert interlinguistique des métaphores terminologiques}

Le poids de la culture partagée dans la formation des métaphores terminologiques est enfin une donnée évidente : comme le remarque Cortès (2003), les processus d'encodage et de décodage métaphoriques présupposent le partage d'un même espace d'interdiscours :

La construction du sens métaphorique présuppose une maîtrise totale de la construction du sens compositionnel. Le sens métaphorique est un construit énonciatif complexe qui mobilise de la part des interlocuteurs des connaissances sur le monde, sur le rapport du locuteur à ce qu'il entend transmettre et à ses interlocuteurs, ainsi que tout ce qu'il sait sur le plan linguistique, y compris sur le sens des unités lexicales 


\begin{abstract}
dans tous leurs emplois, c'est-à-dire avec tous leurs effets de sens en fonction du contexte. Ces connaissances du matériau lexical et de ses emplois reposent sur la connaissance des textes que le locuteur a entendus et analysés et qui l'influencent dans ses choix d'expression. Nous appellerons ce type de compétence l'accès à l'interdiscours. (Cortès, 2003, p.35)
\end{abstract}

En dépit de la prétendue objectivité du langage scientifique et technique, telle que préconisée par Wüster dans ses ouvrages fondateurs de la théorie traditionnelle de la terminologie, les plus grandes inventions terminologiques de l'histoire de la pensée scientifique sont des actes de création langagière (Montuschi, 1993), en ce qu'elles mettent en jeu les ressources de l'imaginaire collectif, indissolublement lié à la culture d'appartenance :

[...] même si les différents domaines du savoir et de la pratique sont souvent les mêmes d'un pays à l'autre, les façons de les découper et de les dénommer ne sont pas les mêmes, en vertu de la relative autonomie du langage. Dumarsais notait déjà que « chaque langue a des métaphores particulières, qui ne sont point en usage dans les autres langues ; par exemple, les Latins disaient d'une armée, dextrum et sinistrum cornu [la corne droite et la corne gauche], et nous disons l'aile droite et l'aile gauche »(1998: 144-145, cité par Gardes-Tamine, 2007, p.15).

Pour ne citer qu'un exemple célèbre (Humbley, in Cortès 2003) le terme du langage informatique bootstrap (qui renvoie à l'expression anglaise to lift/hoist yourself up by your own bootstraps et qui évoque par analogie le fonctionnement de l'application) est lié à une métaphore de la langue commune qui ne présente aucun équivalent conceptuel dans les autres langues; ce lien avec la culture de départ rend la métaphore indissoluble de son contexte linguistique de production, comme en témoigne son histoire récente: adapté dans d'autres langues sous des formes abrégées (boot en italien et en français), ce terme a perdu rapidement ses liens avec la métaphore de départ, devenant un terme opaque à tous les effets pour les non-anglophones. De même, l'exemple du splicing dans le domaine du génie génétique (Humbley, in Cortès 2003) témoignerait selon Temmerman (2000) d'un imaginaire collectif des scientifiques américains fortement influencé par la passion du cinéma et des vidéos; nous pourrions ajouter à ces exemples d'autres exemples plus récents, parmi lesquels la théorie des cordes de Veneziano (1968), ou encore la crêpe stellaire évoquée par l'astrophysicien Jean-Pierre Luminet (voir Giaufret, Rossi, 2013).

L'ancrage culturel de la métaphore dans toutes ses réalisations langagières, particulièrement évident dans le terminologies spécialisées, s'avère d'autant plus délicat si l'on considère les enjeux économiques, sociopolitiques, épistémologiques au sens large, liés aux dynamiques d'implantation terminologique (Humbley, in Cortès 2003) : la langue d'expression terminologique dominante dans un secteur donné témoigne d'une primauté économique et technologique de la langue/culture d'origine des termes et concepts reconnus par la communauté des spécialistes dans le secteur en question. Ce phénomène n'est pas sans retombées sur les politiques linguistiques (voir à ce propos, entre autres et à titre d'exemple, Depecker 2001 ou - pour la situation italienne - Beccaria 2008).

En 2005, John Humbley mettait en évidence à ce propos une lacune importante dans les études en traductologie :

La question de la traduction des métaphores est un sujet très peu abordé dans le contexte des langues de spécialité. [...] On peut s'en étonner, car la place de la métaphore dans les discours et le lexique spécialisés fait l'objet de nombreuses études récentes, englobant des points de vue linguistique, terminologique et surtout cognitif. On sait par ailleurs que la métaphore tient une place importante en tant que technique de création terminologique dans l'aménagement linguistique, en particulier en français. (p.49) 
La traduction des métaphores, objet d'études dans le domaine de la traduction littéraire, est parfois moins étudiée dans les ouvrages de traductologie technique. Sur les termes de spécialité, l'hypothèque d'objectivité postulée par la théorie traditionnelle de la terminologie a encore un poids considérable : conçus comme de simples étiquettes, les termes entretiennent en principe un rapport de correspondance 1:1 d'une langue à l'autre, indépendamment de tout ancrage culturel. Cette conception du terme comme dénomination purement objective engendre l'impression fallacieuse (voir encore une fois Humbley, 2005) que le transfert des termes spécialisés d'une langue de départ à une langue d'arrivée soit un processus de simple transcodage.

En réalité, les approches terminologiques plus récentes, de la socioterminologie de Gaudin (2003), à la terminologie sociocognitive de Temmerman (2000), focalisent leur attention sur l'aspect culturel des termes (voir aussi à ce propos les essais de Diki-Kidiri, Temmerman et Desmet dans le numéro spécial des "Cahiers du Rifal" consacré à la variation terminologique, 2007). Les termes de spécialité, comme tous les éléments d'un système linguistique donné, sont soumis à l'influence du prisme de la culture qui les a générés : la conceptualisation passe par la culture d'appartenance, ce qui n'est pas sans conséquence dans les dynamiques de normalisation terminologique, comme le souligne Temmerman (2007) dans son étude du vocabulaire anglais du génie génétique identifié à une pellicule cinématographique.

La prise en considération du système qui sous-tend le tout n'est pas sans conséquence pour les descriptions terminologiques (plurilingues) et la représentation des connaissances. Reconnaître la force créatrice du système de la langue compte tenu du caractère socialement et culturellement situé de cette dernière peut avoir un impact sur la gestion de ressources terminologiques dynamiques capables de rendre compte de l'évolution du sens (Temmemrman, 2007, p.83)

L'ancrage culturel s'avère par conséquent d'importance capitale dans les processus de transfert des terminologies d'une communauté à l'autre, en ce qu'il mobilise les implicites et les interdiscours partagés, qui peuvent différer sensiblement dans les langues/cultures de départ et d'arrivée. Cet aspect décidera également du succès ou de l'échec de l'implantation des termes dans la langue/culture d'arrivée, d'autant plus pour ce qui est des termes à base métaphorique :

Les mécanismes du succès ou de l'insuccès des néologismes terminologiques dans des cultures différentes, mais partageant une même langue restent encore mal connus et font appel à des études linguistiques orientées vers les habitudes culturelles de chaque société. (Desmet, 2007, p.3)

Dans la pratique de la communication spécialisée, toutefois, cet aspect n'est pas toujours pris suffisamment en compte, notamment dans le transfert de l'anglais (qui reste encore la langue/culture d'origine de la plupart des terminologies scientifiques et techniques) vers les langues romanes, à savoir dans la formation secondaire de terminologies:

By secondary term formation, Sager is alluding to the way concepts conceived and named in one language are named in another language. "Secondary term formation occurs when a new term is created for a known concept [...] as a result of knowledge transfer to another linguistic community" (Sager $1990: 80$ ). In the modern world, where English dominates scientific and technical research, this means the way English-language terms are transposed into other languages. Since the dominance of 
English in this respect seems to be gaining ground, the importance of secondary term formation may be expected to increase. (Humbley, in Cortès, 2003)

L'importance glottopolitique de l'anglais à cet égard est parfois écrasante; dans le cas du transfert des termes à base métaphorique, qui nous intéressent dans le cadre de cette étude, elle engendre dans certains cas la perte totale de l'ancrage analogique sous-jacent aux termes, capital dans le processus d'interaction conceptuelle déclencheur de la phase de décodage et d'appropriation de la métaphore. L'exemple de la théorie des cordes de Veneziano est emblématique à cet égard (voir entre autres Musacchio et Corbolante, 2012) : le terme, conceptualisé en italien sous forme de teoria delle corde (renvoyant à l'analogie entre le branes de l'univers et les cordes d'un violon), passe ensuite en anglais sous forme de string theory, terme qui popularise et rend célèbre la théorie; malheureusement, ce même terme est ensuite retraduit en italien par le calque teoria delle stringhe, qui ne peut qu'engendrer un parallèle avec les chaussures et qui ne renvoie plus aucunement à l'analogie avec le domaine musical. L'analogie est perdue et le terme risque de devenir opaque, voire - comme dans ce cas - fourvoyant pour les usagers. De même, l'option de l'emprunt intégral (bien souvent privilégié en italien) produit une perte cognitive pour les locuteurs de la langue d'arrivée : les termes de l'informatique à base métaphorique en sont un exemple évident, comme en témoignent mouse, bug, et d'autres dénominations à risque d'opacité pour les italophones.

Dans ce contexte, différents cas de figure sont possibles, le plus simple étant le partage total de l'isotopie métaphorique dans les diverses langues/cultures (comme dans le cas cité par Humbley, 2005, des virus informatiques en français, anglais, allemand). Dans d'autres cas, l'uniformité est loin d'être une condition satisfaite ; un exemple tiré du langage de la finance présente des typologies de transfert diverses :

1. partage et explicitation du mécanisme métaphorique sous-jacent la terminologie spécialisée - comme il ressort de l'exemple suivant, où le terme anglais est comparé avec ses hétéronymes en français de France, de Belgique, de Suisse, du Québec et en italien²:

\begin{tabular}{|l|l|l|l|l|l|l|}
\hline Terme de & $\begin{array}{l}\text { Domaines de } \\
\text { la métaphore }\end{array}$ & FRANCETERME & BELTERME & TERMDAT & GDT & AIEBB \\
\hline One-shot & $\begin{array}{l}\text { Finances- } \\
\text { armes à feu }\end{array}$ & Coup unique & Coup unique & $\emptyset$ & Coup unique & $\varnothing$ \\
\hline Floating & $\begin{array}{l}\text { Finances- } \\
\text { navigation }\end{array}$ & Flottant & Flottant & Flottant & Flottant & $\begin{array}{l}\text { Floating } \\
\text { (emprunt } \\
\text { intégral) } \\
\text { Flottante } \\
\text { (calque) }\end{array}$ \\
\hline Burning cost & $\begin{array}{l}\text { Finances- } \\
\text { combustion }\end{array}$ & Taux de flambage & $\varnothing$ & $\varnothing$ & $\begin{array}{l}\text { Taux de } \\
\text { flambage }\end{array}$ & Ø \\
\hline
\end{tabular}

2. Dans d'autres cas, l'isotopie métaphorique subit un processus d'adaptation à la langue/culture d'arrivée, par le passage à une isotopie plus proche de la culture partagée des locuteurs de la langue d'arrivée, comme dans les exemples suivants : 


\begin{tabular}{|c|c|c|c|c|c|c|}
\hline $\begin{array}{l}\text { Terme de } \\
\text { départ }\end{array}$ & $\begin{array}{l}\text { Domaines de la } \\
\text { métaphore }\end{array}$ & FRANCETERME & BELTERME & TERMDAT & GDT & AIEBB \\
\hline $\begin{array}{l}\text { Hit-and- } \\
\text { run }\end{array}$ & Finances-baseball & $\begin{array}{l}\text { Action éclair (dans } \\
\text { ce cas, la } \\
\text { métaphore sportive } \\
\text { est effacée au profit } \\
\text { d'une métaphore } \\
\text { météorologique, } \\
\text { visant la rapidité de } \\
\text { l'action) }\end{array}$ & Action éclair & $\varnothing$ & Action éclair & $\varnothing$ \\
\hline Hot money & Finances-température & $\begin{array}{l}\text { Capitaux flottants / } \\
\text { capitaux fébriles } \\
\text { (la métaphore } \\
\text { d'arrivée met } \\
\text { l'accent sur } \\
\text { l'instabilité de } \\
\text { capitaux, voire sur } \\
\text { leur nature } \\
\text { dangereuse par le } \\
\text { synonyme fébriles } \\
\text { qui partage } \\
\text { toutefois le } \\
\text { domaine de la } \\
\text { température avec la } \\
\text { métaphore } \\
\text { originaire) }\end{array}$ & $\begin{array}{l}\text { Capitaux } \\
\text { flottants / } \\
\text { capitaux } \\
\text { fébriles }\end{array}$ & $\begin{array}{l}\text { Capitaux } \\
\text { fébriles } \\
\text { capitaux } \\
\text { spéculatifs }\end{array}$ & $\begin{array}{l}\text { Capitaux } \\
\text { flottants / } \\
\text { capitaux } \\
\text { fébriles }\end{array}$ & $\begin{array}{l}\text { Hot money } \\
\text { (emprunt } \\
\text { intégral) } \\
\text { syn. Denaro } \\
\text { caldo } \\
\text { (calque) }\end{array}$ \\
\hline $\begin{array}{l}\text { Aggressive } \\
\text { accounting }\end{array}$ & $\begin{array}{l}\text { Finances- } \\
\text { psychologie }\end{array}$ & $\begin{array}{l}\text { Comptabilité } \\
\text { flatteuse } \\
\text { (modulation d'état } \\
\text { d'âme) }\end{array}$ & $\varnothing$ & $\begin{array}{l}\text { Comptabilité } \\
\text { flatteuse }\end{array}$ & $\begin{array}{l}\text { Comptabilité } \\
\text { flatteuse } \\
\text { syn. } \\
\text { Comptabilité } \\
\text { créative }\end{array}$ & $\varnothing$ \\
\hline $\begin{array}{l}\text { Cherry } \\
\text { picking }\end{array}$ & $\begin{array}{l}\text { Finances-activités } \\
\text { productives }\end{array}$ & $\begin{array}{l}\text { Picorage (la } \\
\text { métaphore renvoie } \\
\text { dans ce cas à } \\
\text { l'action des } \\
\text { oiseaux) }\end{array}$ & Picorage & $\varnothing$ & Picorage & $\varnothing$ \\
\hline Tombstone & $\begin{array}{l}\text { Finances- } \\
\text { anthropomorphisme }\end{array}$ & $\begin{array}{l}\text { Faire-part de } \\
\text { clôture } \\
\text { remarquer la } \\
\text { dimension tout } \\
\text { particulièrement } \\
\text { française du faire- } \\
\text { part) }\end{array}$ & $\begin{array}{l}\text { Faire-part de } \\
\text { clôture }\end{array}$ & $\begin{array}{l}\text { Faire-part de } \\
\text { clôture }\end{array}$ & $\begin{array}{l}\text { Faire-part de } \\
\text { clôture }\end{array}$ & $\begin{array}{l}\text { Tombstone } \\
\text { (emprunt } \\
\text { intégral) }\end{array}$ \\
\hline
\end{tabular}


3. Enfin, on identifie des cas de perte complète du pouvoir heuristique de la métaphore de départ, soit à cause de la banalisation de la métaphore - éliminée dans la langue d'arrivée - soit à cause d'une stratégie d'emprunt intégral, qui rend opaque et donc inaccessible la métaphore - c'est le cas, entre autres, des bears and bulls intrinsèquement liés à l'imaginaire collectif nord-américain :

\begin{tabular}{|c|c|c|c|c|c|c|}
\hline $\begin{array}{l}\text { Terme de } \\
\text { départ }\end{array}$ & $\begin{array}{l}\text { Domaines de la } \\
\text { métaphore }\end{array}$ & FRANCETERME & BELTERME & $\begin{array}{l}\text { TERMDA } \\
T\end{array}$ & GDT & AIEBB \\
\hline $\begin{array}{l}\text { Bear } \\
\text { market }\end{array}$ & $\begin{array}{l}\text { Finances- } \\
\text { zoomorphisme }\end{array}$ & Marché baissier & Marché baissier & $\begin{array}{l}\text { Marché } \\
\text { baissier }\end{array}$ & $\begin{array}{l}\text { Marché à la } \\
\text { baisse }\end{array}$ & $\begin{array}{l}\text { Bear market } \\
\text { (emprunt } \\
\text { intégral, } \\
\text { accompagn } \\
\text { é de la } \\
\text { glose orso, } \\
\text { mercato } \\
\text { ribassista) }\end{array}$ \\
\hline Bull market & $\begin{array}{l}\text { Finances- } \\
\text { zoomorphisme }\end{array}$ & Marché haussier & Marché haussier & $\varnothing$ & $\begin{array}{l}\text { Marché } \\
\text { haussier }\end{array}$ & $\begin{array}{l}\text { Bear market } \\
\text { (emprunt } \\
\text { intégral, } \\
\text { accompagn } \\
\text { é de la } \\
\text { glose toro, } \\
\text { mercato } \\
\text { rialzista) }\end{array}$ \\
\hline Haircut & $\begin{array}{l}\text { Finances- } \\
\text { anthropomorphisme }\end{array}$ & Marge de sécurité & Marge de sécurité & $\varnothing$ & $\begin{array}{l}\text { Marge de } \\
\text { sécurité }\end{array}$ & $\varnothing$ \\
\hline Bullet & Finances-armes & $\begin{array}{l}\text { Remboursement in } \\
\text { fine }\end{array}$ & $\begin{array}{l}\text { Remboursement } \\
\text { in fine }\end{array}$ & $\varnothing$ & $\begin{array}{l}\text { Remboursem } \\
\text { ent in fine }\end{array}$ & $\begin{array}{l}\text { Bullet } \\
\text { (emprunt } \\
\text { intégral) }\end{array}$ \\
\hline Blue chip & Finances-poker & $\begin{array}{l}\text { Valeur de premier } \\
\text { ordre }\end{array}$ & $\begin{array}{l}\text { Valeur de premier } \\
\text { ordre }\end{array}$ & $\begin{array}{l}\text { Valeur de } \\
\text { première } \\
\text { ordre - syn. } \\
\text { Valeur père } \\
\text { de famille } \\
\text { (à } \\
\text { remarquer } \\
\text { la } \\
\text { modulation } \\
\text { métaphoriq } \\
\text { ue de la LD } \\
\text { à la LA, où } \\
\text { l'accent est } \\
\text { mis sur la } \\
\text { fiabilité des } \\
\text { titres par la } \\
\text { métaphore } \\
\text { de la } \\
\text { famille) }\end{array}$ & $\begin{array}{l}\text { Valeur de } \\
\text { premier ordre }\end{array}$ & $\begin{array}{l}\text { Blue chip } \\
\text { (syn. Titoli } \\
\text { guida) }\end{array}$ \\
\hline
\end{tabular}




\section{Pour conclure}

Nous espérons avoir présenté - par ce bref aperçu - tout l'intérêt de la thématique de la métaphore en terminologie. Trop souvent limitée par le passé à sa dimension de catachrèse isolée à fonction néonymique, la figure métaphorique assume en réalité dans les langues de spécialité une pluralité de fonctions, caractérisées par des statuts sémiotiques complexes et multiformes, ce qui la rend un sujet encore extrêmement riche et digne d'exploration, notamment dans une perspective contrastive et interculturelle.

\section{Bibliographie}

Assal A. (1994). La métaphorisation terminologique, Terminologie et traduction 2, 1994, pp. 235-242.

Bachelard G. (1980). La formation de l'esprit scientifique : contribution à une psychanalyse de la connaissance objective. Paris : Vrin.

Beccaria, G. L. (2008). Per difesa e per amore. La lingua italiana oggi. Milano: Garzanti.

Black M. (1962). Models and metaphors: Studies in language and philosophy. Ithaca: Cornell University Press.

Bowdle B.F, Gentner, D. (2005). The carreer of metaphor, Psychological Review 112, pp. 193-216.

Boyd R. (1979). Metaphor ad Theory Change: What is "Metaphor" a Metaphor for?, in A. Ortony (éd.), Metaphor and Thought. Cambridge: Cambridge University Press, pp.356-408.

Cabré M. T. (1999). La terminología. Representación y comunicación. Una teoría de base comunicativa y otros artículos. Barcelona: Institut Universitari de Lingüística Aplicada, Universitat Pompeu Fabra (Sèrie Monografies, 3)

Collombat I. (2003). Le discours imagé en vulgarisation scientifique : étude comparée du français et de l'anglais, Metaphorik.de 05/2003 (en ligne, www.metaphorik.de/05/collombat.pdf).

Cortès C. (éd. 2003). La métaphore. Du discours général aux discours spécialisés, Cahier du C.I.E.L. 2000-2003.

Desmet I. (2007). Terminologie, culture et société. Éléments pour une théorie variationniste de la terminologie et des langues de spécialité, Les Cahiers du Rifal, 26, décembre 2007, en ligne, pp. 3-13.

Détrie C. (2001). Du Sens dans le processus métaphorique. Paris : Champion.

Diki-Kidiri M. (éd. 2000). Terminologie et diversité culturelle, Les Cahiers du Rifal, 21, juin 2000, en ligne (http://www.rifal.org/cahiers/)

Diki-Kidiri M. (2007). Éléments de terminologie culturelle, Les Cahiers du Rifal, 26, décembre 2007, en ligne, pp. 14-25.

Dury P., Maniez F., Arlin N., Rougemont C. (éds. 2009). La métaphore dans les langues de spécialité. Grenoble : Presses Universitaires de Grenoble.

Gardes Tamine J. (2007). Les métaphores lexicalisées dans la langue et dans les langues de spécialité : un obstacle à la compréhension, in M.-B. Vittoz (éd.), Synergies Italie 3/2007, pp. 13-22.

Gaudin F. (2003). Socioterminologie : une approche sociolinguistique de la terminologie. De Boeck : Bruxelles.

Gaudin F. (2007) Quelques mots sur la socioterminologie, Les Cahiers du Rifal, 26, décembre 2007, en ligne, pp. 2635 .

Giaufret A., Rossi M. (2013) Métaphores terminologiques, circulation des savoirs et contact entre langues, Signes n.10, 2013 - http://www.revue-signes.info/document.php?id=2928 
Giaufret A., Rossi M. (2010). L'année mondiale de l'astronomie: la diversité terminologique et culturelle de l'espace métaphorique, Actes du Colloque GLAT 2010, Lisboa, 17-19 maggio 2010, pp. 131-144.

Humbley J. (2005). La traduction des métaphores dans les langues de spécialité : le cas des virus informatiques, Linx 52,2005 (en ligne).

Jacobi D. (1986). De la recherche à la vulgarisation; itinéraires du texte scientifique. Annales de l'Université de Besançon, Paris : Les Belles Lettres.

Jacobi D. (1999). La communication scientifique ; discours, figures, modèles. Grenoble: Presses Universitaires de Grenoble (coll. Médias \& sociétés).

Kocourek R. (1991). La langue française de la technique et de la science. Vers une linguistique de la langue savante. Wiesbaden : Oscar Brandstetter, 2e édition augmentée, refondue et mise à jour avec une nouvelle bibliographie.

Kuhn T. S. (1979). Metaphor in science in A. Ortony (éd.), Metaphor and Thought. Cambridge: Cambridge University Press, 1979, pp.409-419.

Lakoff G., Johnson M. (1980). Metaphors we live by. Chicago: Chicago University Press.

Montuschi E. (1993). Le metafore scientifiche. Milano: Franco Angeli, 1993.

Montuschi E. (2013). Metafora e linguaggi della scienza, in M. Prandi, A. Giaufret, M. Rossi (éds.). Il ruolo della metafora nella creazione di terminologie. Genova: GUP, pp. 13-24.

Musacchio M. T., Corbolante L. (2012). When are science and technology (in) accessible ? A diachronic study of the poularisation of new terms in physics and information technology, in AAVV., Terminologie : textes, discours et accès aux savoirs spécialisés, GLAT GENOVA 2012, éd. GLAT, Brest, pp.225-238.

Normand C. (1978). Métaphore et concept. Bruxelles : Complexe.

Oliveira I. (2005). La Métaphore terminologique sous un angle cognitif, Meta, Journal des traducteurs, vol. 50, no 4, 2005, pp. 83-104.

Oliveira I. (2009). Nature et fonctions de la métaphore en science. L'exemple de la cardiologie. Paris: L'Harmattan.

Prandi M. (2012). A Plea for Living Metaphors: Conflictual Metaphors and Metaphorical Swarms, Metaphor and Symbol 27, 2, pp.148-170.

Prandi M., Rossi M. (2012). Les métaphores dans la création de terminologie, dans Terminologie : textes. discours et accès aux savoirs spécialisés, 2012, Ed. du GLAT, pp.7-19.

Prandi M., Giaufret A., Rossi M. (éds. 2013). Il ruolo della metafora nella creazione di terminologie. Genova: GUP.

Paissa P. (2013). Rhétorique et traduction : enjeux théoriques et méthodologiques, in M. Rossi (éd.) Repères Dorif n.s. «Traduction, médiation, interprétation» - volet n.1, juin 2013, http://www.dorif.it/ezine/ezine articles.php?id=65

Resche C. (2002). La métaphore en langue spécialisée, entre médiation et contradiction: étude d'une mutation métaphorique en anglais économique, ASp 35-36, 2002, pp.103-119.

Richards, I. A. (1936). The Philosophy of Rethoric. Oxford : Oxford University Press.

Rossi M. (2009). L'emploi de la métaphore comme ressource pour la néologie terminologique : le cas du langage de la dégustation du vin, in P. Dury et al. (éds.) La métaphore en langues de spécialité, Lyon : PUL, pp.199-227.

Stengers I., Schlanger J. (1991). Les concepts scientifiques. Paris : Gallimard.

Schlanger J. (1995). Les métaphores de l'organisme. Paris : L'Harmattan.

Temmerman R. (2000). Toward New Ways of Terminology Description. The Sociocognitive Approach, Amsterdam/Philiadelphie : John Benjamin.

Temmerman R. (2007). Les métaphores dans les sciences de la vie et le situé socioculturel, Les Cahiers du Rifal, 26, décembre 2007, en ligne, pp. 72-81.

Vandaele S. (2004). Approche cognitive de la traduction dans les langues de spécialité : vers une systématisation de la description de la conceptualisation métaphorique, Meta : journal des traducteurs / Meta: Translators' Journal, Volume 50, numéro 2: 415-431. 
Vandaele S., Lubin L. (2005). Approche cognitive de la traduction dans les langues de spécialité : vers une systématisation de la description de la conceptualisation métaphorique, Meta, Journal des traducteurs vol. 50, n.2, 2005, pp. 415-431.

${ }^{1}$ Les réflexions et les exemples suivants ont également fait l'objet d'une étude en langue italienne, voir le volume Prandi, Giaufret, Rossi, 2013.

2 Banques de données utilisées: FRANCETERME (France), BELTERME (Belgique), TERMDAT (Suisse), GDT=Grand Dictionnaire Terminologique (Québec), AIEBB (Italie). 